\title{
15. \\ Mémoire sur la théorie de la chaleur.
}

(Par Mr. G. Libri de Florence.)

\section{Introduction.}

Lorsque l'illustre géomètre; qui. le premier a découvert les lois de la propagation de la chaleur, s'occupa de cette théorie, les physiciens admettaient presque généralement, que le refroidissement des corps s'opère d'après la différence qui passe entre leur température, et celle du milieu environnant. Depuis cette époque MM. Dulong et Petit sont parvenus, pas des expériences délicates et variées, ’̀ découvrir la véritable loi d'après laquelle la chaleur se propage à la surface des corps. Cette loi remarquable, et entièrement différente de celle que $\mathrm{New}$ ton avait énoncée, paraissait devoir exciter l'attention des. analystes, et les engager ì connaitre les modifications. qu'elle introduirait dans les résultats du. calcul: mais on a dû remarquer, que dans les recherches plus récentes qui ont été publiées sur la théorie de la chaleur, on partait toujours de la loi de Newton.

Lorsqu'il s'agit de températures peu élevées, on peut supposer sans orreur: sensible, que le refroidissement s'opère d'après la différence des temperatures; mais il n'en est pas de même dans le problème général, et quoiqu'on ait cru que l'erreur ne devenait apréciable qu'à des températures très-élevées, déjà à la chaleur de l'eau bouillante, on commet une erreur de presque deux degrés du thermomètre centesimal, dans léquation différentielle qui exprime le mouvement de la chaleur; et cette erreur qui affecte à la fois la valeur. de l'inconnue, et la forme sous laquelle elle se trouve dans l'équation différentielle, doit devenir bien plus sensible dans l'intégrale. Il est vrai qu'en partant de là loi. découverte par M. Dulong, les équations que l'on obtient ne peuvent plus. être intégrées en termes finis avec les méthodes connues; mais il ne paraît pas toujours permis dans les problèmes physiques de s'écarter de là nature; pour simplifièr l'analyse qui sert à les résoudre: et d'ailleurs si une première approximation est suffisante pour les inventeurs d'une théorie, il faut que des recherches ultérieures rapprochent d'avantage le calcul de l'expérience. C'est ainsi que 
Newton ayant découvert le système du monde, il a fallu un siècle de recherches pour construire l'édifice dont il avait posé les fondemens.

Dans le mémoire que nous publions à présent, nous nous sommes proposés de déterminer le mouvement linéaire de la chaleur, en partant de la loi du refroidissement découverte par M. Dulong. On sait que cette loi se compose de deux parties, dont l'une exprime la perte de la chaleur, éprouvée par l'effet du rayonnement, et l'autre représente laction du milieu. Or cette seconde partie est sujette à des variations qu'il est très-difficile de soumettre au calcul: pour $\mathbf{y}$ parvenir il faudrait connaître la théorie des mouvemens des fluides élastiques; mais ee problème considéré dans sa généralité surpasse les forces actuelles de l'aualyse. On a supposé, pour surmonter cette difficulté, que les corps dont on voulait connaître les changemens de température, étaient soumis ¿̀ laction d'un courant d'air de densité et de température constantes, qui frappait tous les points de leur surface avec une vitesse uniforme; mais il est aisé de voir limpossibilité de verifier en nature cette hypothèse, de manière qu'on ne peut tirer de la aucun résultat comparable à l'expérience. Pour rapprocher autant qu'il est possible la théorie de l'observation, nous avons dû considérer le mouvement linéaire de la chaleur, dans une armille de petite épaisseur renfermée dans un espace vide, dont l'enceinte est maintenue à une température constante. Ce problème conduit à une équation aux différentielles partielles qui n'est plus linéaire, et qui contient la variable principale sous la forme d'exponentielle. Il n'est plus possible dans ce cas d'intégrer directement l'équation trouvée, et il faut recourir aux méthodes d'approximation. On ne oonnait pas de méthode pour intégrer par approximation les équations aux différentielles partielles: on peut à la verité exprimer leur intégrale en séries, et l'on parvient, à l'aide du théorème de M. Fourier, à sommer ces séries lorsqu'elles dérivent d'équations linéaires à coëfficiens onstans; mais lorsque la série est trop compliquée pour pouvoir en obtenir le terme général, il est impossible de juger de sa convergence, et le problème reste sans solution. Nous savons tâché d'appliquer aux équations aux différentielles partielles, la méthode d'approximation dont on se sert pour les équations différentielles ordinaires. On sait que pour intégrer les équations différentielles qui expriment les mouvemens des corps oélestes, on fait usage de la méthode d'approximation successive, $\mathfrak{a}$ l'aide de laquelle on les réduit à un nombre indé- 
fini d'équations linéaires; mais il arrive que chaque intégration introduit des arcs de cercle qui détruisent l'effet de l'approximation. Les plus grands géomètres ont tâché de vaincre cette difficulté, mais les méthodes qu'ils ont inventées, quoique très-ingénieuses, deviennent souvent impraticables à cause de la longueur excessive des calculs qu'elles demandent. Et d'ailleurs il est très-difficile de s'assurer, que parmi les termes qu'on néglige il n'en existe aucun qui devienne sensible au bout d'un tems très-long: de telle manière que ces méthodes exigent presqu'autant de sagacité pour les appliquer, qu'il fallait de génie pour les décourrir. Toutes ces difficultés paraissent devoir se retrouver dans les équations aux différentielles partielles; cependant si au lieu d'intégrer complètement la première des équations linéaires que nous avons obtenues, pour prendre des intégrales particulières des autres, comme on le fait pour les équations différentielles ordinaires, on commence par prendre des intégrales particulières, des premières équations que l'on veut considérer, et que l'on n'intègre complètement que celle à laquelle on veut arrêter l'approximation, on obtiendra le nombre de fonctions arbitraires qui est nécessaire pour satisfaire à toutes les conditions du problème, et on sera assuré, comme on le démontre directement, de pouvoir éviter toujours les arcs de cercle. Nous avons effectué le calcul que nous venons d'indiquer, sur les deux premières équations linéaires que fournit le problème, et nous avons trouvé une formule qui se compose de celle queM. Fourier avait déjà donnée, et d'un terme de correction multiplié par une petite quantité. En embrassant un plus grand nombre d'équations, on trouverait la même expression, plus des termes multipliés par les puissances ascendantes de la petite quantité par rapport à laquelle on a développé. La méthode que nous venons d'exposer, peut s'appliquer à l'intégration par approximation d'une classe assez étendue d'équations aux différentielles partielles; mais ces recherches ne sauraient trouver place ici, et elles formeront le sujet d'un mémoire particulier.

Parmi les nombreux corollaires que M. Fourier a déduits de son analyse, il en est un fort remarquable qui prouve, qu'après un tems considérable la demisomme des températures de deux points diamétralement opposés dans larmille, forme toujours une quantité constante, et égale à la température moyenne. Ce résultat a été confirmé avec assez de précision par l'expérience, et il était intéressant de voir comment on tirerait la même conséqueuce de la loi découverte par M. Dulong. En partant 
de la température donnée par notre formule nous obtenons le même théorème, et nous démontrons quil dérive également de l'hypothèse de $\mathrm{New-}$ ton, et de la loi observée.

En supposant le refroidissement proportionnel ì la différence des températures, on trouve qu'en plongeant l'extrémité d'une barre de petite épaisseur dans une source constante de chaleur, lorsque l'équilibre des températures se sera établi, la distribution de la chaleur dans la barre pourra être exprimée par une courbe logarithmique. Si l'on part de la loi observée, on obtient une équation différentielle qui n'est plus linéaire, mais qui peut cependant s'intégrer, et dont l'intégrale fait voir que ce n'est que pour des températures très-peu élevées, que l'état permanent de la barre peut se représenter par une courbe logarithmique: lorsque la chaleur augmente, cet état dépendra d'une transcendante elliptique, et en général il sera donné par une transcendante d'une ordre d'autant plus élevé, que la température sera plus grande.

L'intégrale de l'équation différentielle, qui exprime l'état permanent des températures dans une barre très-mince, n'est propre qu'à donner les températures d'une partie de la barre comprise entre deux foyers successifs : cela est évident dans le cas du mouvement linéaire, et tient à ce que l'équation différentielle que l'on a trouvée, ne se vérifie pas aux points qui servent de foyers. Mais lorsquil s'agit d'un corps d'une figure quelconque, qui a été échauffé primitivement par plusieurs foyers situés ì sa surface ou dans son intérieur, il devient difficile de séparer les diverses parties du corps, pour chacume desquelles l'intégrale que la théorie fournit doit se vérifier, et de déterminer les limites au delà desquelles elle donnerait une valeur fautive. Le corps se subdivise alors, par rapport ì son état calorifique, en d'autres corps dont les surfaces de contact jouissent de la propriété du maximum, ou du minimum de température. La détermination de ces surfaces-limites conduit ì trouver un grand nombre de propriétés importantes dans la théorie de la chaleur, comme nous le montrerons dans une autre occasion.

Lorsqu'on cherche à connaître le mouvement de la chaleur dans un corps, on exprime la température d'un point donné en fonction de ses cordonnées, et du tems écoulé; mais pendant que le corps s'échauffe ou so refroidit, toutes ses molécules se déplacent à cause du changement de volume produit par les variations de la température. On a négligé jusqu'ì 
présent, dans'la théorie mathěmatique de ̣la chaleur, l'altẻration du volume des corps; mais ce phénomène, le plus remarquable et le plus constant de tous ceux qui dépendent de la chaleur, ne nous paraît pas de nature à être négligé. En considérant les dilatations linéaires, nous donnons la formule de correction, qui doit servir ầ déterminer les coordonnées du point dont on connait la température.

Nous n’avons traité, dans ce mémoire, que les cas les plus simples de la théorie de la chaleur; mais nous nous proposons de reprendre ce travail dans la suite, et d'appliquer notre analyse a des 'questions plus compliquées.

\section{A n a 1 y s e.}

Si l'on renferme une armille circulaire homogène de petite épaisseur, dans une sphère creuse, qui ne contienne ni air ni aueune autre espèce de gaz, de manière que le centre de l'armille cö̈ncide avec le centre de la sphère, et si le rayon de celle-ci est beaucoup plus grand que le rayon de l'armille, les parois de la sphère étant d'ailleurs entretenues à une température constante quelconque, il résulte du principe de la communication de la chaleur, et de la loi du refroidissement décourerte par M. Dulong, que le mouvement de la ehaleur dans larmille sera exprimé, à très-peu près, par l'équation

$$
\frac{d v}{d t}-\frac{a d^{2} v}{d x^{2}}+c\left(p^{v}-1\right)=0
$$

dans laquelle $v$ exprime l'excès de la température du point que l'on considère, sur la température de l'enceinte; $x$ représente la distance, comptée sur l'armille même, de ce point à l'origine des coordonnées; $t$ est le tems écoulé depuis que l'armille a abandonné l'état initial des températures; et $p, a$ et $c$, sont des constantes dont la première a pour valeur $\stackrel{20}{r}(1,165)$, et les deux autres se déterminent par l'expérience dans chaque cas partieulier.

En effet, l'équation que M. Fourier a trouvée, en supposant que le refroidissement s'opère d'après la différenee des températures, est de la forme

$$
\text { 7. } \frac{d v}{d t}-\frac{a d^{2} v}{d x^{2}}+C v=0 \text {, }
$$

et en y substituant au lieu de $C v$, le terme $c\left(p^{v}-1\right)$ qui exprime la loi du refroidissement dans le vide, d'après les expériences de MM. Dulong et Petit, on aura l'équation 


$$
\text { 8. } \frac{d v}{d t}-\frac{a d^{2} v}{d x^{2}}+c\left(p^{v}-1\right)=0,
$$

que nous avions déjà indiquée.

Avant d'aller plus loin, il convient d'examiner un résultat que lon a obtenu en faisant $v=e^{--c t} u$ dans l'équation (\%.); car par cette substitution elle se transforme dans la suivante

$$
\frac{d u}{d t}-\frac{a d^{2} u}{d x^{2}}=0
$$

qui est la même équation (7.) dans laquelle on a supposé quîl n'y avait aucune déperdition de chaleur à la surface; d'où il résulte que dans l'hypothèse de $\mathrm{New}$ to $\mathrm{n}$, le refroidissement qui s'opère à la surface ne change pas la loi de la distribution de la chaleur. Mais comme il n'est pas possible d'effectuer une réduction semblable sur l'équation (8.), qui dérive de la loi observée, il faudra admettre qu'en nature, même dans le mouvement linéaire, la distribution de la chaleur est troublée par leffet de la déperdition qui a lieu d̀ la surface.

Maintenant si l'on fait $\log p=\delta$, l'équation (8.) prendra la forme

$$
\frac{d v}{d t}-\frac{a d^{2} v}{d x^{2}}+c\left(e^{\delta v}-1\right)=0
$$

l'exposant $\delta=\frac{\mathrm{r}}{2} \mathrm{O} \log \left(\frac{1}{1} \frac{1}{0} \frac{\sigma}{0} \frac{5}{0}\right)$ étant une très-petite quantité; et si l'on développe en série l'exponentielle dans cette équation, on aura

$$
\frac{d v}{d t}-\frac{a d^{2} v}{d x^{2}}+c d v+\frac{c \delta^{2} v^{2}}{1.2}+\frac{c \delta^{3} v^{3}}{1.2 .3}+\text { etc. }=0
$$

et par suite, en faisant $c \delta=b$, on obtiendra

$$
\frac{d v}{d t}-\frac{a d^{2} v}{d x^{2}}+b v+\frac{b \delta v^{2}}{1.2}+\frac{b \delta^{2} v^{3}}{1.2 .3}+\text { etc. }=0
$$

Si lon fait à présent

$$
v=V+\delta V_{2}+\delta^{2} V_{2}+\delta^{3} V_{3}+\text { etc. }
$$

et que lon substitue cette valeur dans l'équation précédente, en ordonnant le résultat par les puissances ascendantes de $\delta$, on trouvera

$$
\text { 9. } \begin{aligned}
0 & =\frac{d V}{d t}-\frac{a d^{2} V}{d x^{2}}+b V+\delta\left(\frac{d V_{1}}{d t}-\frac{a d^{2} V_{1}}{d x^{2}}+b V_{2}+\frac{b V^{2}}{1.2}\right) \\
& +\delta^{2}\left(\frac{d V_{2}}{d t}-\frac{a d^{2} V_{2}}{d x^{2}}+b V_{8}+b V V_{3}+\frac{b V^{3}}{1.2 .3}\right)+\text { etc. }
\end{aligned}
$$

et en égalant â zéro séparément les coëfficiens de chaque puissance de $\delta$, on aura les équations

$$
\frac{d V}{d t}-\frac{a d^{2} V}{d x^{2}}+b V=0,
$$

Crelle's Journal d. M. VII. Bd. 2. Hft. 


$$
\begin{aligned}
& \frac{d V_{1}}{d t}-\frac{a d^{2} V_{1}}{d x^{2}}+b V_{1}+\frac{b V^{2}}{1.2}=0, \\
& \frac{d V_{2}}{d t}-\frac{a d^{2} V_{2}}{d x^{2}}+b V_{2}+b V V_{1}+\frac{b V^{3}}{1.2 .3}=0,
\end{aligned}
$$

dont le nombre sera déterminé par l'exposant de la plus grande puissance de $\delta$, que l'on veut considérer.

En intégrant la première de ces équations, on obtiendra la valeur de $V$ qui étant substituée dans la seconde équation, servira ì déterminer $V_{1}$ et ainsi de suite, en introduisant dans la dernière équation les valeurs des inconnues déduites des équations précédentes, on déterminera une nouvelle inconnue. Mais il faut observer, qu'au lieu de prendre l'intégrale complète de la première équation, pour la substituer dans la seconde, et puis intégrer complètement celle-ci, pour substituer encore la valeur de linconnue dans la suivante, et ainsi de suite, on pourra exprimer

$$
V, V_{1}, V_{2}, V_{3}, \ldots V_{n-1} \text {, }
$$

par des intégrales particulières, et $\delta^{n}$ étant la dernière puissance de $\delta$ que l'on veut considérer, il suffira d'intégrer complètement l'équation multipliéce par $\delta^{n}$, qui comprendra les diflérentielles de $V_{n}$ et les quantités connues $V, V_{1}, V_{2}, \ldots . V_{n-1}$; car lintégrale complète de cette équation, contiendra toutes les fonctions arbitraires, qui sont nécessaires à la résolution générale du problème.

Supposons par exemple que dans l'équation (9.) on veuille avoir égard à la première puissance de $\delta$ seulement, et négliger toutes les autres; on aura les deux équations

$$
\begin{aligned}
& \frac{d V}{d t}-\frac{a d^{2} V}{d x^{2}}+b V=0 \\
& \frac{d V_{x}}{d t}-\frac{a d^{2} V_{1}}{d x^{2}}+b V_{1}+\frac{b V^{2}}{1.2}=0
\end{aligned}
$$

dans la première desquelles on prendra une valeur particulière de $V$, pour la substituer dans la seconde équation, que l'on devra intégrer complètement. Maintenant, on sait que l'on satisfait à l'équation

$$
\frac{d V}{d t}-\frac{a d^{2} V}{d x^{2}}+b V=0
$$

en faisant $V=(\sin n x+\cos n x) e^{-\left(b+a n^{2}\right) t}, n$ étant une constante indéterminée. Si l'on substitue cette valeur de $V$ dans l'équation

$$
\frac{d V_{1}}{d t}-\frac{a d^{2} V_{3}}{d x^{2}}+b V_{1}+\frac{b V^{2}}{1.2}=0
$$


on aura

$$
\begin{aligned}
& \frac{d V_{1}}{d t}-\frac{a d^{2} V_{1}}{d x^{2}}+b V_{2}+\frac{b}{1.2}(\sin n x+\cos n x)^{2} e^{-2\left(b+a n^{2}\right) t} \\
= & \frac{d V_{1}}{d t}-\frac{a d^{2} V_{1}}{d x^{2}}+b V_{2}+\frac{b}{1.2}(1+\sin 2 n x) e^{-2\left(b+a n^{2}\right) t}=0,
\end{aligned}
$$

et en faisant $V_{\mathrm{s}}=y e^{-2\left(b \tan ^{2}\right) t}+Z$ ( $y$ étant fonction de $x$ seulement, et $Z$ fonction de $x$ et de $t$ ), on obtiendra, après avoir divisé par $e^{-2\left(b+a n^{2}\right) t}$, $2\left(b+a n^{2}\right) y+\frac{a d^{2} y}{d x^{2}}-b y-\frac{b}{1.2}(1+\sin 2 n x)-\frac{d Z}{d t}+\frac{a d^{2} Z}{d x^{2}}-b Z=0$, et si lon égale à zéro séparement les termes qui contiennent $Z$, on aura, après les réductions, les deux équations

$$
\begin{gathered}
\frac{d^{2} y}{d x^{2}}+\left(\frac{b}{a}+2 n^{2}\right) y=\frac{b}{2 a}(1+\sin 2 n x), \\
\text { 10. } \quad \frac{d Z}{d t}-\frac{a d^{2}}{d x^{2}}+b Z=0,
\end{gathered}
$$

dont la première a pour intégrale

$$
y=\left\{\begin{array}{c}
\left(E_{1}+\frac{b}{2 a V /\left(\frac{b}{a}+2 n^{2}\right)} \int d x(1+\sin 2 n x) \sin x V\left(\frac{b}{a}+2 n^{2}\right)\right) \cos x V\left(\frac{b}{a}+2 n^{\prime}\right) \\
+\left(E_{2}-\frac{b}{2 a V\left(\frac{b}{a}+2 n^{2}\right)} \int d x(1+\sin 2 n x) \cos x V\left(\frac{b}{a}+2 n^{2}\right)\right) \sin x V /\left(\frac{b}{a}+2 n^{2}\right)
\end{array}\right.
$$

et par suite on obtiendra

$r=E_{2} \cos y V\left(\frac{b}{a}+2 n^{2}\right)+E_{2} \sin x V\left(\frac{b}{a}+2 n^{2}\right)-\frac{b}{2}\left(\frac{1}{b+2 a n^{2}}+\frac{\sin 2 n x}{b-2 a n^{2}}\right)$.

Si l'on intègre à présent l'équation en $Z$, on aura

$$
Z=\left(a_{p} \sin p x+b_{p} \cos p x\right) e^{-\left(b+a p^{2}\right) t}
$$

$a_{p}, b_{p}$ et $p$, étant des quantités quelconques; et comme l'équation (10.) est linéaíre et ne contient pas de terme indépendant de $Z$, il s'ensuit qu'elle sera satisfaite par une somme de termes semblables à la valeur de $Z$ que nous avons déji trouvée, pourviu que les constantes $a_{p}, b_{p}, p$, soient différentes. Et par conséquent l'intégrale complète de l'équation (10.) sera composé d'une suite infinie de valeurs semblables à celle que nous avons déjà obtenue, pourvu que l'on change convenablement les constantes arbitraires.

Maintenant, puisque $Z$ contient une infinité de constantes arbitraires, on pourra dans la valeur de $y$ supprimer les deux constantes $E_{1}, E_{2}$, qui ne rendraient pas plus généralo la valeur de $\mathcal{F}_{1}$; aloes en fuisant 
$E_{1}=E_{2}=0$, on aura une valeur de $y$ qui ne contiendra plus les fonctions $\cos x V\left(\frac{b}{a}+2 n^{2}\right) ; \quad \sin x V\left(\frac{b}{a}+2 n^{2}\right)$.

Soit $r$ le rayon de l'armille dans laquelle on suppose que la chaleur se propage; sa circonférence sera égale à $2 r \pi$, et il est clair qu'en exprimant par $v$ la température d'un point dont la distance à l'origine est $x$, la valeur de $v$ ne devra pas changer lorsque dans la formule qui exprime $v$ on mettra $x+2 r \pi$, à la place de $x$ : par conséquent il faudra, dans les valeurs de $V$, et de $V_{2}$, faire $n=\frac{m}{r}$, et $p=\frac{s}{r} ; m$ et $s$ étant deux nombres entiers positifs quelconques: on aura donc, en négligeant les puissances supérieures de $\delta$,

$$
=\left\{\begin{array}{c}
v=V+\delta V_{1}=V+\delta\left(y e^{-2\left(b+a n^{2}\right) t}+Z\right) \\
\left(\sin \frac{m x}{r}+\cos \frac{m x}{r}\right) e^{-\left(b+\frac{\alpha m^{2}}{r^{2}}\right) t}+\sum_{s=0}^{s=\infty}\left(a_{s} \sin \frac{s x}{r}+b_{s} \cos \frac{s x}{r}\right) \delta e^{-\left(b+\frac{a s^{2}}{r^{2}}\right) t} \\
-\frac{b r^{2}}{2}\left(\frac{1}{b r^{2}+2 a m^{2}}+\frac{\sin \frac{2 m x}{r}}{b r^{2}-2 a m^{2}}\right) \delta e^{-8\left(b+\frac{a m^{2}}{r^{2}}\right) t}
\end{array}\right.
$$

mais le premier terme $\left(\sin \frac{m x}{r}+\cos \frac{m x}{r}\right) e^{-\left(b+\frac{\alpha m^{2}}{r}\right) t}$ de cette formule pourra être compris sous le signe $\Sigma$, puisque la valeur de $m$ devra se trouver parmi les valeurs de $s$; et en faisant $\delta a_{s}=A_{s}$, et $\delta b_{s}=B_{s}$, on aura

$$
\text { 11. } v=\left\{\begin{array}{c}
\sum_{s=0}^{s=\infty}\left(A_{s} \sin \frac{s x}{r}+B_{s} \cos \frac{s x}{r}\right) e^{-\left(b+\frac{a s^{2}}{r^{2}}\right) t} \\
-\frac{b r^{2}}{2} \delta\left(\frac{1}{b r^{2}+2 a m^{2}}+\frac{\sin \frac{2 m x}{r}}{b r^{2}-2 a m^{2}}\right) e^{-2\left(b+\frac{a m^{2}}{r^{2}}\right) t}
\end{array}\right.
$$

et cette formule exprimera le mourement de la chaleur dans l'armille. Pour déterminer la fonction arbitraire, ou, ce qui revient au même, la série des termes

$$
\begin{gathered}
A_{1}, A_{2}, A_{3}, \ldots \ldots A_{s} \text {, etc., } \\
B_{1}, B_{2}, B_{3}, \ldots \ldots B_{s} \text {, etc., }
\end{gathered}
$$

on devra faire $t=0$, et on aura $v$ égal à la température initiale, que nous exprimerons par la fonction $f(x)$, et qui étant développée en série suivant les sinus et cosinus des multiples de l'arc $\frac{x}{r}$ servira pour déterminer les coëfficiens.

Si dans la formule (11.) on fait $\delta=0$, on obtiendra l'expression que M. Fourier a trouvée le premier en partant de l'hypothèse de $\mathrm{New-}$ 
ton. Pour déterminer $m$, on devra prendre le nombre entier le plus petit qui ne satisfait pas ì l'équation

$$
b r^{2} \pm 2 a m^{2}=0
$$

puisqu'on aura de cette manière la valeur la plus approchée, et on sera assuré de ne pas rencontrer des arcs de cercle qui détruiraient l'effet de lapproximation. Lorsque les conditions du problème permettront de faire $m=0$, le terme de correction, pour la première approximation, se réduira $\dot{a}-\frac{\delta}{2} e^{-8 b t}$; et on trouvera que ce terme est indépendant des coordonnées, et quil ne dépend que du tems.

On a vu qu'en faisant $E_{1}=E_{2}=0$, on obtenait

$$
\begin{array}{r}
y=\frac{b}{2 a \sqrt{ }\left(\frac{b}{a}+2 n^{2}\right)}\left\{\begin{array}{r}
\cos x \sqrt{ }\left(2 n^{2}+\frac{b}{a}\right) \int d x(1+\sin 2 n x) \sin x \sqrt{ }\left(2 n^{2}+\frac{b}{a}\right) \\
-\sin x \sqrt{ }\left(2 n^{2}+\frac{b}{a}\right) \int d x(1+\sin 2 n x) \cos x \sqrt{ }\left(2 n^{2}+\frac{b}{a}\right)
\end{array}\right\} \\
=-\frac{b}{2}\left(\frac{1}{b+2 a n^{2}}+\frac{\sin 2 n x}{b-2 a n^{2}}\right)
\end{array}
$$

de manière que les fonctions $\cos x\left(\frac{b}{a}+2 n^{2}\right), \sin x V\left(\frac{b}{a}+2 n^{2}\right)$, s'évanouissaient d'elles-mêmes dans le calcul: il était nécessaire que cette réduction pût s'effectuer, autrement la température $v$ ne serait pas restée la même en changeant $x$ en $x+2 r \pi$, dans la formule qui la représente. Cependant cette réduction, qui a paru un résultat de calcul, aura toujours lieu quel que soit le nombre des puissances de $\delta$ que l'on considère; en effet on a toujours l'équation

$$
=-\frac{\varphi(x)}{a}-\frac{1}{a^{2}}\left(\cos a x \int d x \frac{d^{2} \cdot \varphi(x)}{d x^{2}} \sin a x-\sin a x \int d x \frac{d^{2} \cdot \varphi(x)}{d x^{2}} \cos a x\right)
$$

$=\frac{(-1)^{p}}{a^{2 p}}\left(\cos a x \int d x \frac{d^{2 p} \cdot \varphi(x)}{d x^{2} p} \sin a x-\sin a x \int d x \frac{d^{2 p} \cdot \varphi(x)}{d x^{2 p}} \cos a x\right)$

$$
-\frac{\varphi(x)}{a}+\frac{d^{2} \cdot \varphi(x)}{a^{3} d x^{2}}-\frac{d^{4} \cdot \varphi(x)}{a^{6} d x^{4}} \ldots+\frac{(-1)^{p}}{a^{2 p-1}} \frac{d^{2 p-2} \cdot \varphi(x)}{d x^{2 p-2}}
$$

qui montre, que si entre $\varphi(x)$, et $\frac{d^{2 p} \cdot \varphi(x)}{d x^{3 p}}$ on peut avoir une équation de la forme $M \varphi(x)=\frac{d^{2 p} \cdot \varphi(x)}{d x^{2 p}}, M$ étant une quantité constante, on pourra toujours avoir

$$
\begin{aligned}
& \text { 12. } \cos a x \int d x \varphi(x) \sin a x-\sin a x \int d x \varphi(x) \cos a x \\
& =\left(\frac{a^{q p}}{a^{2 p}-(-1)^{p} M}\right)\left(-\frac{\varphi(x)}{a}+\frac{d^{2} \cdot \varphi(x)}{a^{3} d x^{2}} \ldots .+\frac{(-1)^{p}}{a^{q p-2}} \frac{d^{q p-q} \cdot \varphi(x)}{d x^{2 p-2}}\right)
\end{aligned}
$$


et l'on voit que le second membre ne contiendra ni $\cos a x$, ni $\sin a x$. Il est clair que si $\varphi(x)=A \sin m x+B \cos m x$, on pourra établir l'équation $\frac{d^{2} \varphi(x)}{d x^{2}}=-m^{2} \varphi(x)$, et par conséquent on obtiendra la valeur de l'intégrale (12.) délivrée de $\sin \alpha x$, et de $\cos \alpha x ;$ la même chose arrivera en général lorsque $\varphi(x)$ sera de la forme

$$
\begin{array}{r}
A_{1} \sin m_{1} x+A_{2} \sin m_{2} x \ldots+A_{t} \sin m_{t} x+\text { etc. } \\
+B_{1} \cos m_{1} x+B_{2} \cos m_{2} x \ldots+B_{t} \cos m_{t} x+\text { etc. }
\end{array}
$$

et lorsqu'on aura

$$
\varphi(x)=a+b x+c x^{2}+d x^{3} \ldots . .+e x^{n}
$$

et dans d'autres cas.

Maintenant, puisque les valeurs particulières de $V, V_{1}, V_{2}$, etc., peuvent toujours s'exprimer par des fonctions de la forme

$$
e^{-l t}\left\{\begin{array}{r}
P_{2} \sin n_{1} x+P_{2} \sin n_{2} x \ldots+P_{q} \sin n_{q} x \\
+Q_{2} \cos n_{1} x+Q_{2} \cos n_{2} x \ldots+Q_{q} \cos n_{q} x
\end{array}\right\}
$$

on sera toujours assuré que les sinus et cosinus des arcs irrationnels ne se trouveront pas dans l'intégrale complète, quel que soit le nombre des puissances de $\delta$ que l'on voudra considérer.

Il faut observer que si l'on avait $(-1)^{p} M-a^{2 p}=0$, le second membre de l'équation (12.) aurait une valeur infinie: on rencontrerait cette circonstance si l'un des nombres $n_{1}, n_{2}, n_{3}, \ldots n_{q}$, etc., compris dans les valeurs de $V, V_{1}, V_{2}$, etc., était égal à $a$; alors en reprenant le calcul on trouverait, après les réductions, un terme de la forme $N x \sin n_{q} x$, qui contiendrait l'arc de cercle $x$, et qui rendrait nul, dans le cas que nous considérons, l'effet de l'approximation: mais il est clair que le nombre $m$ de la formule (11.) pourra toujours être déterminé de manière que cela n'arrive pas, et il suffira à cet effet que le dénominateur ne soit pas zéro, comme nous l'avons déjà indiqué.

La formule (12.) exige, pour être appliquée facilement, que la fonction $\varphi(x)$ puisse se réduire à une suite finie de monomes composés dun seul facteur: cependant en poussant fort loin lapproximation, et en calculant un grand nombre de termes de la serie $V+\delta V_{2}+\delta^{2} V_{3}+$ etc., on aurait en général

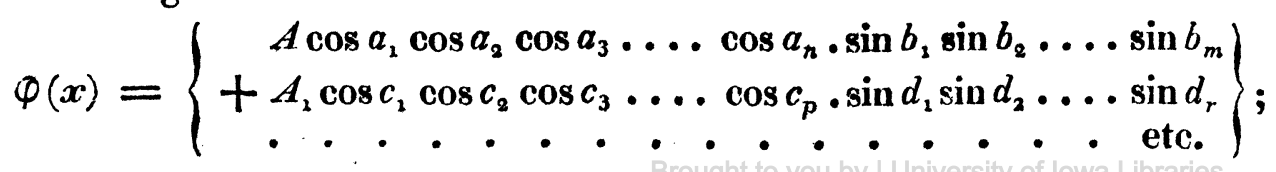


pour opérer les réductions nécessaires dans cette formule on pourra faire usage de réquation

$$
=\frac{1}{2^{n-1}}\left(\cos q+S \cdot \cos \left(q-2 a_{y}\right)+S \cdot S \cdot \cos \left(q-2 a_{y}-2 a_{z}\right) \ldots+\text { etc. }\right),
$$

$$
\cos a_{1} \cos a_{2} \cos a_{3} \ldots \ldots \cos a_{n}
$$

dans laquelle les ares $a_{1}, a_{2}, a_{3}, \ldots a_{n}$, sont indéterminés et tous différens entre eux, et donnent $a_{2}+a_{2}+a_{3} \ldots+a_{r}=q$; en indiquant par $S \cdot \cos \left(q-2 a_{y}\right)$, la somme de tous les termes de la forme $\cos \left(q-2 a_{y}\right)$, où l'on a fait successivement $y=1,2,3, \ldots n$ : en représentant par $S . S \cdot \cos \left(q-2 a_{y}-2 a_{z}\right)$, la somme de tous les termes de la forme $\cos \left(q-2 a_{y}-2 a_{z}\right)$, où lon a fait d'abord successivement $y=1,2,3, \ldots \ldots n$; et où l'on a donné $\mathfrak{i} z$ toutes les valeurs $1,2,3, \ldots . n$ (pourvu que lon néglige tous les termes dans lesquels on aurait $y=z$ ); et ainsi de suite juscuau dernier terme: en observant toujours d'omettre tous les termes de cette formule, qui seraient égaux ì lun de ceux que l'on a déjì trouvés. En différentiant cette équation par rapport à $a_{1}, a_{2}, a_{3}$, etc., on obtiendrait des expressions semblables, pour le produit d'un nombre quelconque de sinus et de cosinus.

M. Fourier en adoptant la loi de Newton a trouvé, que la demisomme des températures de deux points de larmille situés aux extrémités Gin ciancire qualconque, forme toujours, au bout d'un temps très-long, une quantité constante, et égale ì la température moyenne de larmille. Maintenant, puisque ce résultat à été confirmé par l'expérience, il est clair quil devra se déduire encore de la loi du refroidissement découverte par MM. Dulong et Petit. En effet, en reprenant la valeur de $v$ trouvée précédemment (11.), et en $y$ supposant $t$ très-grand, on devra considérer seulement deux espèces de termes: ceux dans lesquels on a $s=0$, et ceux qui résultent de $s=1$ : c'est-ì-dire les termes multipliés par $e^{-b t}$, et par $e^{-\left(b+\frac{a}{r^{2}}\right) t}$; puisque tous les autres qui contiennent quelques-uns des facteurs $e^{-8\left(b+a \frac{m^{2}}{r^{2}}\right) t} ; e^{-\left(b+4 \frac{a}{r^{2}}\right) t}$ etc.; sont trop petits (dans lhypothèse de $t$ très-grand) pour être comparés ì cenx-ci. Alors la valeur de $v$ se réduira à la forme

$$
v=B_{0} e^{-b t}+A_{2} e^{-\left(b+\frac{a}{r^{2}}\right) t} \sin \frac{x}{r}+B_{2} e^{-\left(b+\frac{a}{r^{2}}\right) t} \cos \frac{x}{r}
$$

et il est clair que si dans cette équation on substitue $x+r \pi$, au lieu de $x$, on aura la température $v_{1}$, du point de l'armille diamétralement opposé à celui dont la distance à lorigine est $x$, exprimée de cette manière 
et partant

$$
v_{1}=B_{0} e^{-b t}-A_{1} e^{-\left(b+\frac{a}{r^{2}}\right) t} \sin \frac{x}{r}-B_{2} e^{-\left(b+\frac{a}{r^{2}}\right) t} \cos \frac{x}{r}
$$

$$
\frac{v+v_{x}}{2}=B_{0} e^{-b t}
$$

Si dans la formule (11.) on substitue pour $b$ sa valeur $c \delta$, on trouvera, que l'expression de $M$. F o urier contient seulement la première puissance de $\delta$, et que la nôtre renferme aussi $\delta^{2}$. Avec notre méthode, on pourra pousser l'approximation aussi loin que l'on voudra, sans crainte de rencontrer jamais des arcs de cercle qui la rendraient nulle; et on pourra toujours déterminer les constantes arbitraires (que l'on trouve en prenant des integrales particulières des premières équations différentielles, pour les substituer dans celles qui suivent) de manière que tous les termes aillent toujours en décroissant, et qu'aucun dénominateur ne s'évanouisse; comme nous l'avons fait dans l'analyse précédente.

On sait que l'équilibre des températures dans une armille, dont un point est soumis à une température constante, est donné lorsqu'on adopte la loi de Newton, par l'équation

$$
\frac{d^{2} v}{d x^{2}}=n^{2} v
$$

dont lintegrale est $\nu=A e^{n x}+A_{1} e^{-n x}, A$ et $A_{1}$ étant deux constantes arbitraires. En partant de la loi de M. Dulong on obtient, pour léquilibre des températures dans le vide, l'équation

qui a pour intégrale

$$
\frac{d^{2} v}{d x^{2}}=a\left(e^{\delta v}-1\right)
$$

$$
x=\int \frac{d v}{\sqrt{\left(2 a\left(\frac{e^{\delta v}}{\delta}-v\right)+C-\frac{2 a}{\delta}\right)}}+C_{1} ;
$$

$C$ et $C_{1}$ étant deux nouvelles constantes arbitraires. Lorsque $v$ est une petite quantité, on peut supposer sans erreur sensible

$$
x=\int \frac{d v}{r\left(C+a \delta v^{2}\right)}+C_{1}=\frac{1}{V(a \delta)} \log \left(v V\left(\frac{a \delta}{C}\right)+V\left(1+\frac{a \delta v^{2}}{C}\right)+C_{1},\right.
$$

et en faisant $C_{1} V(a \delta)=\log E ; a \delta=n^{2}$; et réduisant, on aura

$$
\left(\frac{e^{n x}}{E}-\frac{n v}{V(C)}\right)^{2}=1+\frac{n^{2} v^{2}}{C}=\frac{e^{2 n x}}{E^{2}}-\frac{2 n v e^{n x}}{E V(C)}+\frac{n^{2} v^{2}}{C},
$$

et par suite

$$
v=\frac{e^{n x} V(C)}{2 n E}-\frac{E V(C)}{2 n e^{n x}}=A e^{n x}+A_{1} e^{-n x} ;
$$


en faisant $A=\frac{V(C)}{2 n E} ; A_{2}=-\frac{E V(C)}{2 n} ;$ et comme $v$ est égal à la température $t$ du point que l'on considère, moins la température $T$ de l'enceinte, on obtiendra enfin l'équation

$$
t=A e^{n x}+A_{1} e^{-n x}+T ;
$$

qui dans le cas de $T=0$, coïncide avec celle que nous avions déjà trouvée, en supposant le refroídissement proportionnel à la différence des températures.

Si l'on considère une barre indéfinie très-mince, et que l'on suppose un foyer de température constante placé sur cette barre ì l'origine des coordonnées, lorsque l'équilibre des températures se sera établi, cet état sera représenté par l'équation $\frac{d^{2} v}{d x^{2}}=n^{2} v$, pourvu que la température $v$ soit assez petite pour pouvoir négliger, dans le développement de $\boldsymbol{e}^{\delta v}$, les puissances de $\delta v$ supérieures à la première: maintenant on sait que l'intégrale de léquation précédente est $v=C e^{n x}+C_{2} e^{-n x}$; mais comme d'ailleurs l'état permanent de la barre est exprimé depuis $x=-\infty$, jusqu'ì $x=+\infty$, par l'équation

$$
v=C_{2}\left(\frac{2}{\pi} \int_{0}^{x} \frac{d q \cos q x}{1+q^{2}}\right)^{n},
$$

et que quelque valeur que l'on attribue aux constantes, ces deux expressions de $v$ ne peuvent jamais cö̈ncider, il en résulterait que lintégrale de léquation $\frac{d^{2} v}{d x^{2}}=n^{2} v$, qui est linéaire, pourrait se former de la somme des deux valeurs de $v$ que nous venons de rapporter, et contiendrait troís constantes arbitraires. Pour expliquer ce paradoxe il est nécessaire d'observer que l'équation $\frac{d^{2} v}{d x^{2}}=n^{2} v$, n'exprime l'état permanent de la barre, que dans les points qui permettent un flux de chaleur du point qui précède immédiatement celui que l'on considère, à celui-ci, et de celui-ci, à l'autre qui le suit immédiatement; tandis que le foyer, que nous avons placé à l'origine des coordonnées, envoye un flux continuel de chaleur ì tous les points de la barre qui sont situés ì sa droite et à sa gauche. Le même résultat se déduirait de la considération de la figure, qui exprime les valeurs des températures pour chaque point de la barre, et dont l'équation est 


$$
y=C_{2}\left(\frac{2}{\pi} \int_{0}^{\infty} \frac{d q \cos q x}{1+q^{2}}\right)^{n} ;
$$

car pour $x=0$, au lieu de donner $\frac{d^{2} y}{d x^{2}}=n^{2} C_{2}$, comme cette courbe devrait faire si elle satisfaisait dans tous ses points â l'équation $\frac{d^{2} y}{d x^{2}}=n^{2} y$, on voit, par la construction, qu'elle donne $\frac{d^{2} y}{d x^{2}}=\infty$; puisque la courbe dont nous parlons est formée de deux demi-logarithmiques égales, qui se réunissent de manière à avoir leur tangente commune au point de contact, perpendiculaire à l'axe des abscisses. Les mêmes considérations s'appliquent à une barre dont plusieurs points sont entretenus à des températures constantes: elles sont très-simples, mais nous avons cru devoir les placer ici, comme étant propres à limiter l'étendue que l'on serait tenté d'attribuer aux éfruations différentielles du mouvement de la chaleur, ou à leurs intégrales. Il faudra surtout $\mathbf{y}$ avoir égard, lorsqu'on voudra connaître l'état calorifique d'un corps, dont un ou plusieurs points pris dans son intérieur ou à sa surface, sont supposés des foyers de temperatures invariables.

Une autre observation que nous croyons ne pas devoir omettre, e'est que lorsque dans l'armille circulaire, que nous avons considérée, nous sommes partis de la propriété connue, que la temperature du point $x$ devait être égale à celle du point dont la distance à l'origine est $x+2 r \pi$, pour déterminer la forme des fonctions circulaires comprises dans l'intégrale, nous lavons fait en suivant l'exemple des illustres géomètres qui nous ont précédé dans ce genre de recherches. Cependant il paraît, qu'au lieu de partir de cette considération auxiliaire, on auraît dû déterminer les coëfficiens de l'arc $x$ d'après l'équation qui exprime la figure de l'armille, et l'irradiation qui se fait intérieurement entre les diverses parties de lanneau; irradiation qui est modifiée, comme l'on sait, d'après la courbure intérieure de l'armille. Ceci deviendrait surtout évident, si l'on dovait déterminer le mouvement de la chaleur sur une surface cylindrique creuse.

Enfin il faut remarquer, que la théorie mathématique de la chaleur a pour but de trouver à chaque instant la température d'un point queloonque, d'après les conditions initiales du problème et la figure du corps que l'on considère. Cependant, dans la solution de ce problème, 
on suppose toujours que les coordonnées du point en question n'ont point changé, pendant que le corps s'est échauffé ou refroidi ; quoiqu'il soit certain que ce point a changé de position, d'après l'augmentation ou la diminution de volume que le corps a soufferte par l'action de la chaleur. En opérant de cette manière on trouve la température d'une molécule matérielle, exprimée en fonction des coordonnées du point qu'elle occupait dans l'espace au commencement du phénomène, et du tems écoulé depuis la cessation de létat initial: pour corriger les formules que l'on a obtenues, il faut connaître la loi d'après laquelle les corps se dilatent par l'effet de la chaleur. Si l'on suppose les dilatations linéaires élémentaires, proportionnelles aux accroissemens de la température, ce qui paraît toujours permis, du moins entre certaines limites de l'échelle thermométrique, on trouvera que le point qui, lorsque la température du corps était zéro, avait $x$ pour distance à lorigine des coordonnées, sera éloigné de l'origine de la quantité $x_{1}=\int d x(1+a v) ; v$ étant donné en fonction de $x$ et de $t$; et le coëfficient a étant donné dans chaque cas par l'expérience. Mais ceci n'est qu'un aperçu, que nous reprendrons dans une autre circonstance.

Ce mémoire est le mème, à quelques changemens près, qui a été lu en 1825 al l'Acadímie Royale des Sciences de Paris. 